\title{
Skin Test and Blastogenic Responses to
}

\section{Sporotrichum schenckii}

\author{
Russell W. Steele, Preston B. Cannady, Jr., William L. Moore, Jr., and \\ LAYNe O. Gentry \\ From the Section of Infectious Diseases, Departments of Medicine and \\ Pediatrics, Brooke Army Medical Center, Fort Sam Houston, Texas 78234
}

\begin{abstract}
A в S T R A C T In vivo skin testing and in vitro lymphocyte blastogenesis were evaluated in a young adult population as methods for detecting cellular immunity to Sporotrichum schenckii. Similar procedures for Candida albicans and Coccidioides immitis were also investigated. 5 of 143 subjects had positive skin tests and 14 had positive blastogenic responses to $S$. schenckii. These 14 subjects also exhibited unusually high responses to $C$. albicans in vitro and 11 of the 14 were female. Data demonstrated a correlation coefficient of 0.89 when comparing the blastogenic assays for $S$. schenckii and $C$. albicans, suggesting cross antigenicity.

Intact cellular immune mechanisms in combination with exposure to $C$. albicans may protect the host from systemic infection with $S$. schenckii. Although a limited number of subjects were studied, as a group, females had more vigorous cellular immune responses to $C$. albicans than males. The rare occurrence of sporothrix infection in females as compared to males may be the result of antigenic stimulation from commonly observed vaginal colonization with $C$. albicans. The present data indirectly support this hypothesis.
\end{abstract}

\section{INTRODUCTION ${ }^{1}$}

Although accidental trauma with inoculation of the fungus, Sporotrichum schenckii, probably accounts for most cases of cutaneous-lymphatic sporotrichosis, systemic disease may be primarily the result of inadequate

Dr. Moore's present address is: Forest Hill Veterans Administration Hospital, Augusta, Ga. 30904. Dr. Gentry's present address is: Baylor College of Medicine, Texas Medical Center, Houston, Tex. 77025.

Received for publication 1 April 1975 and in revised form 8 September 1975.

${ }^{1}$ The opinions or assertions contained herein are the private ones of the authors and are not to be interpreted as official or reflecting the view of the Department of the Army. host immune defense. The overwhelming male predominance cannot be explained by outdoor exposure alone and the rarity in children, but predisposition among immunosuppressed hosts and alcoholics also suggests that endogenous host factors may be of major import $(1,2)$.

The immune mechanisms involved in preventing or combating systemic sporotrichosis are incompletely understood but apparently include both humoral and cellular responses since patients with defects in antibody production or cellular immunity may be predisposed. The relative importance of the cellular immune response has not been well defined, but the severe and often fatal infections of patients with cancer and those on immunosuppressive therapy, both conditions known to depress cellular immune competence, suggest that cellular immunity may provide primary host defense. Moreover, fungal infections such as systemic candidiasis have been reported in children with thymic deficiency syndromes $(3,4)$.

The present study was undertaken to develop an in vitro assay of cellular immunity to $S$. schenckii and to compare this assay to in vivo skin testing. The in vitro method employed was lymphocyte blastogenesis in response to the sporothrix antigen. The application of these assays for diagnosis of a patient with articular sporotrichosis is also presented. In addition, similar assays of cellular immunity to Candida albicans and Coccidioides immitis were evaluated in adult males and females to delineate any cross-reactivity between these agents which might then account for the apparent protection of females from infection with $S$. schenckii.

\section{METHODS}

Lymphocyte blastogenesis. Lymphocyte reactivity to the three fungal agents was evaluated by determining lymphocyte blastogenesis during incubation with the fungal antigens (5). Briefly, lymphocytes were separated from 4-5 $\mathrm{ml}$ of peripheral whole blood by a modification of the 
Hypaque-Ficoll gradient technique (6). The cell suspension was washed twice in culture medium and the lymphocytes were adjusted to a concentration of $2 \times 10^{8} / \mathrm{ml}$ in RPMI 1640 medium with Hepes buffer, containing $100 \mathrm{U}$ of penicillin per $\mathrm{ml}$ and $100 \mu \mathrm{g}$ of streptomycin per $\mathrm{ml}$ and $20 \%$ autologous serum. In some experiments, fetal calf serum and AB-positive serum were also employed. Using a biopipet. $0.1 \mathrm{ml}$ of the lymphocyte suspension $\left(2 \times 10^{5}\right.$ lymphocytes) was mixed with an equal volume of fungal antigen material in one well of sterile flat-bottom microtest plates. All cultures were prepared in triplicate and three dilutions of each fungal antigen were included along with control cultures containing lymphocytes incubated with medium alone. The plates were covered with sterile plastic lids and cultures were incubated for 5 days. $24 \mathrm{~h}$ before harvest, $0.05 \mathrm{ml}$ of RPMI 1640 medium containing $0.1 \mu \mathrm{Ci}$ of [2${ }^{14} \mathrm{C}$ ] thymidine was added to each well. A harvesting apparatus previously described (7) was employed for the separation of reacting cells on glass-fiber filters, for washing of these cells, and for recovery of the radioactive material incorporated by stimulated lymphocytes. The glass-fiber disks were then dried in an oven and transferred to vials containing $5 \mathrm{ml}$ of scintillation fluid for counting in a Beckman Liquid Scintillation Spectrometer (Beckman Instruments, Inc., Fullerton, Calif.). The average counts per minute $(\mathrm{cpm})$ of triplicate samples were determined and results were expressed as a blastogenic index $(\mathrm{BI})^{2}$ : cpm of $\left[{ }^{14} \mathrm{C}\right]$ thymidine uptake for lymphocytes incubated with antigen divided by uptake following incubation with medium alone :

$$
\mathrm{BI}=\frac{\mathrm{cpm} \text { for lymphocytes }+ \text { antigen }}{\mathrm{cpm} \text { for lymphocytes }+ \text { medium }} \text {. }
$$

Fungal antigens. S. schenckii antigen used in the in vitro assay of lymphocyte blastogenesis was prepared from the mycelial phase of the fungus which had been originally isolated from a patient with systemic sporotrichosis. The organisms was grown on Sabouraud's glucose agar at $30^{\circ} \mathrm{C}$. The organism was harvested, washed three times with tissue culture medium and homogenized in a Dounce homogenizer. Large particles were allowed to settle, after which the supernate was collected and serial dilutions subsequently tested for antigenic reactivity as described above. Approximately $200 \mathrm{mg}$ per $\mathrm{ml}$ of antigenic material constituted the stock preparation. This material was later heat inactivated at $56^{\circ} \mathrm{C}$ for $60 \mathrm{~min}$ after it was demonstrated that this process did not alter the sensitivity or specificity of the assay. A dilution of 1:200 was the optimal concentration for most positive responders.

Material for skin testing to $S$. schenckii was kindly supplied by Dr. Leo Kaufman of the Mycoses Immunology Unit, Center for Disease Control, Atlanta, Ga. The yeast phase of the organism was grown on brain-heart infusion broth (Difco Laboratories, Detroit, Mich.) at $37^{\circ} \mathrm{C}$ and shaken at $150-160 \mathrm{rpm}$. The preparation was treated with 1: 10,000 thimerosal, filtered to remove the whole yeast cells and dialyzed against distilled water.

C. albicans antigen used both for skin testing and in vitro study was obtained from Hollister-Stier Laboratories, Inc., Yeadon, Pa., as Dermatophytin "O" undiluted, and C. immitis antigen was obtained as a commercial lot from The Cutter Laboratories, Berkeley, Calif. These preparations were dialyzed six times against 200 vol of $0.15 \mathrm{M} \mathrm{NaCl}$ for $48 \mathrm{~h}$ to remove preservatives. Material was stored at

${ }^{2}$ Abbreviations used in this paper: BI, blastogenic index; FBS, fetal bovine serum. $-20^{\circ} \mathrm{C}$ until used. For skin testing, a $1: 100$ dilution of the original preparation of $C$. albicans and a $1: 10$ dilution of $C$. immitis were employed. Maximum in vitro stimulation of lymphocytes was obtained with a $1: 2,000$ dilution of $C$. albicans and 1:5,000 dilution of $C$. immitis.

Skin testing. Skin test material was applied in the usual manner by injecting $0.1 \mathrm{ml}$ intradermally on the volar surface of the forearm. Results were measured at 24, 48, and $72 \mathrm{~h}$ with the maximum response recorded. A skin test was considered positive $(+)$ if the amount of induration was $10 \mathrm{~mm}$ or greater for $C$. albicans and $5 \mathrm{~mm}$ or greater for $S$. schenckii and $C$. immitis. Erythema was recorded but not considered of importance when the final data were analyzed.

Test subjects. 143 young, healthy adults, 18-39-yr-old, were evaluated for their skin test (in vivo) reactivity and lymphocyte blastogenic (in vitro) responses to the three fungal antigens. These subjects included 87 female and 56 male volunteers. Most were students or hospital personnel. The average age for males and females was similar $(27 \pm 4$ yr vs. $25 \pm 3 \mathrm{yr})$ and none had histories of sporothrix infection or significant contact with gardens or nurseries.

Study case. A 58-year-old Caucasian male with articular sporotrichosis was evaluated with the assays outlined. His diagnosis was confirmed by recovery of the organism from joint fluid. Serum antibody to $S$. schenckii was $1: 256$ by tube agglutination and 1:32 by latex agglutination.

$\mathrm{He}$ had noted the gradual onset of pain in his left knee $2 \mathrm{yr}$ before admission but was not evaluated until $1 \mathrm{yr}$ after onset of symptoms when $\mathrm{X}$ rays revealed a cystic lesion of the left patella. The patella was removed and found to have granulomatous changes but no organisms were found by culture or by special fungal stains. 2 mo after operation drainage from the incision site occurred from which $S$. schenckii was cultured. The subject was treated with potassium iodide for $3 \mathrm{mo}$, but shortly thereafter swelling and pain recurred and aspiration of the left knee joint revealed $S$. schenckii on culture. Therapy with Amphotericin B was begun.

\section{RESULTS}

Data for skin testing and in vitro assays of lymphocyte blastogenesis are summarized in Table I. Results for each of the three fungal antigens were similar in that control subjects whose skin tests were positive also always had positive in vitro blastogenic reactions; however, occasional donors with negative skin tests had positive in vitro assays. More specifically, 21 control subjects $(14.7 \%)$ had negative skin tests to $C$. albicans but a $\mathrm{BI}>3$ to the same antigen.

C. albicans. As summarized in Table I, a higher percentage of women than men had both positive skin tests, 86 vs. $73 \%$ and blastogenic indices, 99 vs. $91 \%$. The mean BI was also higher in women, $15.5 \pm 8.4$, as compared to $6.7 \pm 3.4$ for male control subjects; this difference was statistically significant $(P<0.05)$.

$S$. schenckii. The mean BI to $S$. schenckii in the study population was $1.08 \pm 0.78$. Therefore a BI of three or greater was considered positive. Four female and one male subject had positive skin tests to $S$. schenckii and 11 females and 3 males had a $\mathrm{BI}>3$. Positive skin tests ranged from $6-10 \mathrm{~mm}$. Fig. 1 includes all subjects with 
TABLE I

Skin Test (In Vivo) and Lymphocyte Blastogenic (In Vitro) Reactions to C. albicans, S. schenckii, and C. immitis in a Young Adult Population

\begin{tabular}{|c|c|c|c|c|c|c|c|}
\hline & \multirow[b]{2}{*}{ Subjects } & \multicolumn{2}{|c|}{ C. albicans } & \multicolumn{2}{|c|}{ S. schenckii } & \multicolumn{2}{|c|}{ C. immitis } \\
\hline & & $\underset{\text { test }}{(+) \text { Skin* }}$ & $(+)$ BIf & $\begin{array}{c}(+) \text { Skin } \\
\text { test }\end{array}$ & $(+) B I$ & $\begin{array}{c}(+) \text { Skin } \\
\text { test }\end{array}$ & $(+) B I$ \\
\hline & $n$ & $\%$ & $\%$ & $\%$ & $\%$ & $\%$ & $\%$ \\
\hline Total & 143 & $116(81)$ & $137(96)$ & $5(3)$ & $14(10)$ & $4(3)$ & $4(3)$ \\
\hline Female & 87 & $75(86)$ & $86(99)$ & $4(5)$ & 11 (13) & $1(1)$ & 1 (1) \\
\hline Male & 56 & $41(73)$ & 51 (91) & $1(2)$ & $3(5)$ & $3(5)$ & $3(5)$ \\
\hline
\end{tabular}

* A positive reaction $(+)$ indicates induration of $10 \mathrm{~mm}$ or greater for C. albicans and $5 \mathrm{~mm}$ for $S$. schenckii and $C$. immitis.

$\ddagger$ A blastogenic index (BI) of 3 or greater was considered positive $(+)$.

a BI for $C$. albicans of $>15.0$ and demonstrates that the donors with a positive BI for $S$. schenckii were those with unusually high blastogenic responses to $C$. albicans. The data presented in Fig. 1 exhibited a correlation coefficient of 0.89 for blastogenic responses to candida as compared to sporothrix. This correlation is interpreted as being highly significant.

C. immitis. Four donors, three male and one female, had both positive skin tests and in vitro blastogenic responses to $C$. immitis. The average BI for these four donors was 6.1. None of these had positive results with $S$. schenckii but all were also positive in vivo and in vitro to $C$. albicans. The reactions to candida were not unusually high.

The assays were also repeated substituting fetal bovine serum (FBS) and $A B$-positive antibody-negative serum for autologous serum. No significant alteration was observed in the blastogenic assays. FBS frequently appeared to stimulate significantly the lymphocyte cultures, giving high background counts and thus obviating meaningful interpretation of results.

Study case. Data from the patient with articular sporotrichosis are summarized in Table II. Compared to the test group, this patient was the only one we have

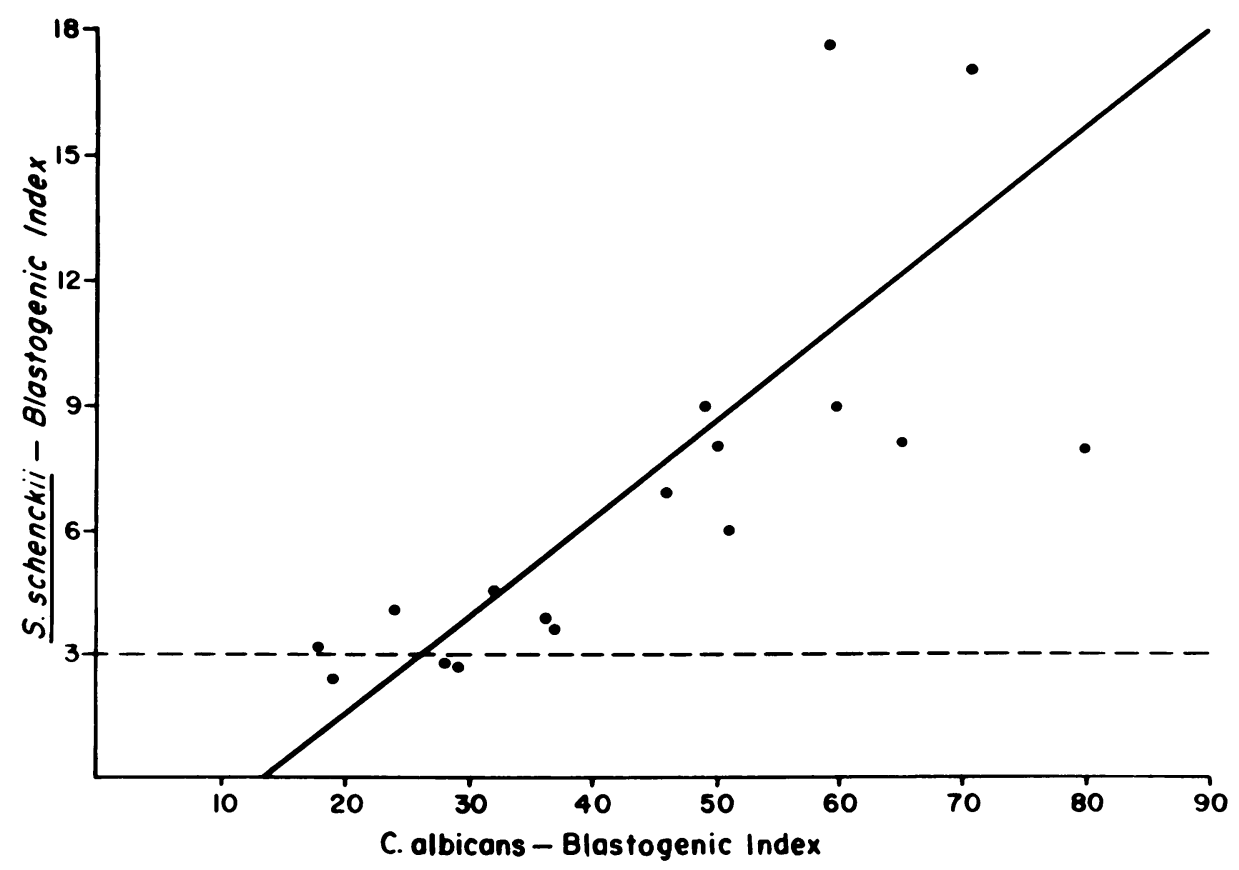

FIGURE 1 Correlation of the blastogenic index for $S$. schenckii with the blastogenic index for $C$. albicans in a normal young adult population. The straight solid line is the calculated linear regression line $(r=0.89)$. 
studied whose skin test was positive and whose BI to $S$. schenckii was higher than the BI to $C$. albicans. There were two test subjects with a $\mathrm{BI}>17.1$, the determination for this patient; but in both instances the response to $C$. albicans was strikingly high as seen in Fig. 1.

Other patients we have studied have included those with systemic candidiasis and systemic coccidioidomycosis. None of these patients have thus far exhibited positive responses to $S$. schenckii. Such cases, however, usually represented immune deficiency states.

\section{DISCUSSION}

$S$. schenckii is an uncommon cause of systemic infection in the United States as well as most parts of the world, and this rarity has certainly discouraged careful investigation into the natural history and pathogenesis of the organism. The epidemic outbreak including almost 3,000 gold mine workers in Witwatersrand, South Africa, 3 decades ago strikingly demonstrated the potential hazards of the fungus, and this mycosis does remain endemic among workers in South Africa, France, Mexico, and perhaps among nursery workers in many countries $(1,2)$. Moreover, the incidence of systemic infection appears to be increasing, especially in the immunologically compromised host (1). In previous reviews of systemic sporotrichosis $(1,2)$ one-half of reported cases were observed in patients with underlying malignant disease.

Present methods of diagnosis include primarily fungal culture and serological methods. For cutaneous-lymphatic sporotrichosis, fungal cultures offer the most direct and reliable laboratory procedure, but cultures are often negative in systemic disease since adequate specimens are difficult to obtain. For this reason, the clinician must usually rely on other methods of diagnosis. Serological assays, including slide latex agglutination, tube agglutination, complement fixation, immunodiffusion, and indirect fluorescent antibody techniques are available in a few research laboratories and, depending on the assay employed, are capable of detecting between 56 and $94 \%$ of the cases of sporotrichosis $(8,9)$. Reports indicate that these methods are specific and reliable. However, there is increasing evidence that development of cellular immunity precedes antibody production (10), and thus tests of cellular immunity to $S$. schenckii may detect infection earlier in the course of the disease. Early detection is, certainly, most essential when ministering to debilitated or immunologically compromised patients. In this clinical setting, immune responses may be somewhat decreased, but complete absence of reactivity in assays like those presently considered should not occur except in severe or terminal states.

This report evaluated in vivo and in vitro methods of detecting reactivity to $S$. schenckii using a skin test
TABLE II

Skin Test and Lymphocyte Blastogenic Reactions of a Patient with Articular Sporotrichosis

\begin{tabular}{lcr}
\hline Organism & Skin test* & \multicolumn{1}{c}{ BI } \\
\hline & $m m$ & \\
C. albicans & 30 & 8.3 \\
S. schenckii & 15 & 17.1 \\
C. immitis & Negative & 1.4 \\
\hline
\end{tabular}

* Millimeter of induration.

and an assay of lymphocyte blastogenesis. The skin test has been investigated previously and demonstrated to be a useful diagnostic tool $(11,12)$. In one study (12) $11 \%$ of the control population had positive skin test responses as compared to $58 \%$ in a population of nursery workers who had at least $10 \mathrm{yr}$ of exposure to plants and soil. No information is available from this study as to responses to $C$. albicans skin test antigen. There were no differences with sporothrix according to sex. In vitro assays of cellular immunity to $S$. schenckii have not been previously reported. It should be noted that blastogenic reactivity, particularly, low level responses, may represent $\mathrm{B}$ cell stimulation. Although there is no evidence to date that $B$ cells respond to the antigens employed in the present report, further investigation is needed to confirm this assay as indicating only cellular immune ( $T$ cell) reactivity.

The present study confirms the sensitivity of the skin test material, as only 5 of the 143 control subjects developed a positive reaction while a patient with articular sporotrichosis had a strongly positive response. Similarly, the in vitro blastogenic assay appeared to be a useful diagnostic procedure, as results for the study case were also positive and higher than responses to other fungal antigens employed. However, further studies on other patients with systemic sporotrichosis are certainly needed to confirm the applicability of these diagnostic tests.

Of great interest is the cross-reactivity between $C$. albicans and $S$. schenckii as demonstrated in the assays of lymphocyte blastogenesis. This is most probably on the basis of antigens common to the two mycotic agents which contribute to development of the cellular immune response. Such cross-reactivity has also been observed in previous serological studies when the whole yeast agglutination assay was employed $(13,14)$. Other serological methods have not demonstrated similar crossreactivity $(8,9)$ suggesting that either the assays of lymphocyte blastogenesis and yeast agglutination are more sensitive or that different antigens are responsible for stimulating some antibody responses.

Most reported cases of sporotrichosis have occurred in males and the observed cross-reactivity between 
C. albicans and S. schenckii may in part account for the lower incidence of infection in females. A recent report (1) indicated that only 1 of 26 patients with systemic sporotrichosis was a female. Even in children there has been a male predominance (15) for sporothrix infection further implying protection in the female host early in life.

The present laboratory data demonstrate that virtually all normal females have positive in vitro responses (i.e. a $\mathrm{BI}>3$ ) to $C$. albicans and that their responses are significantly higher than those of normal male donors. A higher percentage of females than males also have positive in vitro responses to $S$. schenckii but this appears most related to an increased sensitivity to $C$. albicans (Fig. 1).

It is well recognized that $C$. albicans is a frequent inhabitant of the female vaginal tract, thus providing persistent antigenic stimulation during much of life. Males certainly do not carry this mycotic agent as commonly and it is not surprising, therefore, to observe less consistent and lower cellular immune responses to $C$. albicans. Other groups we have evaluated who have exhibited decreased skin sensitivity and lymphocyte blastogenesis to $C$. albicans have included patients with malignancy and those on immunosuppressive therapy; these patients are also ones observed to have a greater predisposition to sporothrix infection $(1,2)$.

In conclusion, the present data indirectly support the hypothesis that intact cellular immune mechanisms in combination with exposure to the fungus $C$. albicans protect the host from systemic infection with $S$. schenckii; however such cross resistance remains to be proven. Many aspects of sporothrix infection have yet to be adequately defined, particularly the role of environmental exposure to $S$. schenckii and the relative importance of antibody production. There is still no direct evidence that cell-mediated immunity protects against sporotrichosis. Sporotrichosis is, of course, too uncommon for any one medical center to accumulate meaningful data, so such information can best be provided by combining the experience of many centers.

Because present therapy for systemic sporotrichosis must include the highly toxic antifungal antibiotic agent, amphotericin B, a better understanding of host defense mechanisms may direct advances in improving management. If cellular immunity is of major importance, as is suggested by our present understanding of fungal dis- ease, then enhancement of these immune responses with agents like levamisole or with transfer factor may provide a beneficial adjunct to current therapy.

\section{ACKNOWLEDGMENTS}

This work was supported in part by research grant no. C-14-74 from the Clinical Investigation Unit of Brooke Army Medical Center.

\section{REFERENCES}

1. Lynch, P. J., J. J. Voorhees, and E. R. Harrell. 1970. Systemic sporotrichosis. Ann. Intern. Med. 73: 23-30.

2. Wilson, D. F., J. J. Mann, J. E. Bennett, and J. P. Utz. 1967. Clinical features of extracutaneous sporotrichosis. Medicine (Baltimore). 46: 265-279.

3. Quie, P. G., and R. A. Chilgren. 1971. Acute disseminated and chronic mucocutaneous candidiasis. Semin. Hematol. 8 : 227-242.

4. Steele, R. W., C. Limas, G. R. Thurman, M. Schuelein, H. Bauer, and J. A. Bellanti. 1972. Familial thymic aplasia. N. Engl. J. Med. 270 : 1295-1301.

5. Bloom, B. R., and P. R. Glade. 1971. In Vitro Methods in Cell-Mediated Immunity. Academic Press, Inc., New York. 443 pp.

6. Thorsby, E. 1967. Cell specific and common antigens on human granulocytes and lymphocytes demonstrated with cytotoxic hetero-antibodies. Vox Sang. 13: 194-206.

7. Steele, R. W., S. A. Hensen, M. M. Vincent, D. A. Fuccillo, and J. A. Bellanti. 1973. A ${ }^{51} \mathrm{Cr}$ microassay technique for cell-mediated immunity to viruses. J. Immunol. 110: 1502-1510.

8. Blumer, S. O., L. Kaufman, W. Kaplan, D. W. McLaughlin, and D. E. Craft. 1973. Comparative evaluation of five serological methods for the diagnosis of sporotrichosis. Appl. Microbiol. 26: 4-8.

9. Roberts, G. D., and H. W. Larsh. 1971. The serologic diagnosis of extracutaneous sporotrichosis. Am. J. Clin. Pathol. 56: 597-600.

10. Steele, R. W., S. A. Hensen, M. M. Vincent, D. A. Fuccillo, and J. A. Bellanti. 1974. Cellular immune responses to rubella following immunization. J. Infect. Dis. 130 : $449-453$.

11. Schneidau, J. D., Jr., L. M. Lamar, and M. A. Hairston. 1964. Cutaneous hypersensitivity to sporotrichin in Louisiana. J. Am. Med. Assoc. 188: 121-123.

12. Ingrish, F. M., and J. D. Schneidau, Jr. 1967. Cutaneous hypersensitivity to sporotrichin in Maricopa county, Arizona. J. Invest. Dermatol. 49: 146-149.

13. Welsh, R. D., and C. T. Dolan. 1973. Sporothrix whole yeast agglutination test: low-titer reactions of sera of subjects not known to have sporotrichosis. Am. J. Clin. Pathol. 59: 82-85.

14. Karlin, J. V., and H. S. Nielsen, Jr. 1970. Serologic aspects of sporotrichosis. J. Infect. Dis. 121 : 316-327.

15. Orr, E. R., and H. D. Riley, Jr. 1971. Sporotrichosis in childhood: report of ten cases. J. Pediatr. 78: 951957. 